\title{
Control of Blood Pressure in Chronic Kidney Disease: How Low to Go?
}

\author{
Anadil Faqah Tazeen H. Jafar \\ Section of Nephrology, Department of Medicine, Aga Khan University, Karachi, Pakistan
}

\section{Key Words}

Chronic kidney disease $\cdot$ Blood pressure $\cdot$ Diabetic nephropathy $\cdot$ Non-diabetic nephropathy $\cdot$ Albuminuria . Proteinuria

\begin{abstract}
Blood pressure (BP) lowering is an important therapeutic goal in patients with diabetic and non-diabetic chronic kidney disease (CKD) for slowing progression and preventing onset of cardiovascular disease. The guidelines for treatment of hypertension in patients with CKD recommend a target $\mathrm{BP}<130 / 80 \mathrm{~mm} \mathrm{Hg}$, with no clear threshold on the lower limit. However, results of recent randomized controlled trials on CKD indicate that aggressive lowering of BP may not provide additional benefit in the vast majority of patients. This paper will review the literature on the main trials examining the question concerning the optimal level of target $\mathrm{BP}$ in patients with CKD and also discuss reasonable target BP levels in light of the evidence, as well as future direction for research in such patients.

Copyright $\odot 2011$ S. Karger AG, Basel
\end{abstract}

Chronic kidney disease (CKD) is a major public health challenge [1]. National estimates from the United States indicate about 13 million people are affected with CKD, with over 650,000 people on renal replacement therapy and with considerable financial implications on national health spending $[2,3]$. Furthermore, many patients with earlier stages of CKD die from premature cardiovascular disease (CVD) before reaching the stage of requiring renal replacement therapy, leading to loss of productive life years.

Epidemiological data provide robust evidence that high blood pressure (BP) is a risk factor for progression of CKD and premature CVD [4-8]. Lowering BP reduces the risk of progression in both diabetic and non-diabetic CKD. In most trials of BP lowering in patients with CKD, an average of three to four agents were required to achieve good control of BP [9]. However, the use of antihypertensive agents also has implications on potential side effects, costs, and quality of life $[10,11]$. Thus, to what extent should BP be lowered for optimal benefit to prevent progression of CKD, and onset of CVD in patients with CKD has been extensively debated. This paper will review the evidence on the subject.

\section{BP Goal in Diabetic CKD}

The potential benefit of aggressive lowering of BP on diabetic nephropathy was first suggested in an uncontrolled trial on 11 subjects with insulin-dependent diabetes in the early 1980 s by Parving et al. [12], in which a significant reduction in rate of decline in glo-

\section{KARGER \\ Fax +4161306 1234 E-Mail karger@karger.ch} www.karger.com
Prof. Tazeen H. Jafar

Department of Medicine, Aga Khan University

Stadium Road, Karachi 74800 (Pakistan)

Tel. +92 213493 0051, ext. 4812

E-Mail tazeen.jafar@aku.edu 
merular filtration rate (GFR) was observed before the treatment $(0.89 \mathrm{ml} / \mathrm{min} / \mathrm{month})$ to during the treatment $(0.22 \mathrm{ml} / \mathrm{min} / \mathrm{month})$ with a mean achieved BP of $129 / 84 \mathrm{~mm} \mathrm{Hg}$.

Subsequently, four primary randomized controlled trials (RCTs) with BP-lowering intervention in patients with type 2 diabetes aimed to address this question (table 1): the Hypertension Optimal Treatment (HOT) study, United Kingdom Prospective Diabetes Study (UKPDS), the Appropriate BP Control in Diabetes (ABCD) trial, and the Action to Control Cardiovascular Risk in Diabetes (ACCORD) study. In the HOT study, 18,790 subjects with hypertension $(<10 \%$ were diabetics with no specified level of serum creatinine or urine albu$\mathrm{min}$ ) were randomized to three diastolic BP (DBP) target subgroups $(<90,<85$, or $<80 \mathrm{~mm} \mathrm{Hg})$, and the results demonstrated a mortality benefit of lower levels ( $<80 \mathrm{vs}$. $90 \mathrm{~mm} \mathrm{Hg}$ or higher DBP) [13]. However there were no significant differences in serum creatinine levels at the end of the 3.8 years. Moreover, the incidence of major cardiovascular events did not differ in patients with a baseline serum creatinine level $>133 \mu \mathrm{mol} / \mathrm{l}(\mathrm{n}=470)$ among the three BP target groups.

The UKPDS was the first large-scale RCT conducted during the 1980s to determine the impact of intensive $(<150 / 85 \mathrm{~mm} \mathrm{Hg})$ versus conventional $(<180 / 105$ $\mathrm{mm} \mathrm{Hg}$ ) control on the development and progression of CKD due to type 2 diabetes $[14,15]$. Over 8.4 years of follow-up, significant risk reductions in macrovascular (34\%) and microvascular (37\%) outcomes were observed in the intensive BP control group. However, it should be noted that the target $\mathrm{BP}$ goal $(<150 / 80 \mathrm{~mm} \mathrm{Hg})$ and the minimum achieved BP $(144 / 82 \mathrm{~mm} \mathrm{Hg})$ in tight control were higher than the conventional targets recommended in the current JNC-7 guidelines [16]. Thus, the UKPDS was not designed to answer the question of whether reduction of BP to levels $<140 / 90 \mathrm{~mm} \mathrm{Hg}$ would be beneficial.

However, secondary analysis of UKPDS data revealed that each $10 \mathrm{~mm} \mathrm{Hg}$ decrease in mean systolic BP (SBP) was associated with reductions in risk of $12 \%$ for any complication related to diabetes, $17 \%$ for deaths related to diabetes, $11 \%$ for myocardial infarction and $13 \%$ for microvascular complications. Any reduction in BP was likely to reduce the risk of complications, with no threshold level, the lowest risk being in those with SBP $<120$ $\mathrm{mm} \mathrm{Hg}$ [14]. Thus the notion of keeping BP as low as possible in patients with diabetic CKD was recommended, albeit based on post-hoc analysis with inherent limitations.

Control of Blood Pressure in CKD:

How Low to Go?
The ABCD trial also aimed to examine the role of intensive versus moderate $\mathrm{BP}$ reduction on the prevention of CVD-related events, and progression of nephropathy, neuropathy, and retinopathy in individuals with type 2 diabetes [17]. The normotensive group that received intensive therapy to lower BP had a mean BP of $128 \pm$ $0.8 / 75 \pm 0.3 \mathrm{~mm} \mathrm{Hg}$, as compared with a mean BP of 137 $\pm 0.7 / 81 \pm 0.3 \mathrm{~mm} \mathrm{Hg}$ in the group that received standard therapy $(\mathrm{p}<0.001)$. There was no significant change in the primary outcome of mean renal function (as assessed by $24 \mathrm{~h}$ creatinine clearance) between the randomized groups during 5 years. The intensive therapy group had less progression from normoalbuminuria to microalbuminuria $(\mathrm{p}=0.01)$ and from microalbuminuria to overt albuminuria $(p=0.03)$, less progression of diabetic retinopathy $(\mathrm{p}=0.02)$, and a lower incidence of stroke $(p=0.03)$. Thus, while the primary outcomes were inconclusive, the secondary outcomes of the ABCD trial indicated some benefit of lower BP goals. Moreover, while albuminuria at baseline was associated with faster progression of kidney disease and onset of CVD, a differential effect of intensive versus standard levels of BP by levels of albuminuria on these adverse outcomes were not reported.

More recently, in the ACCORD study, a total of 4,733 participants with type 2 diabetes were randomly assigned to intensive therapy, targeting an SBP $<120$ $\mathrm{mm} \mathrm{Hg}$, or standard therapy, targeting an SBP $<140$ $\mathrm{mm} \mathrm{Hg}[18,19]$. Despite the fact that there was a significant and sustained difference in BP between intensive therapy $(119.3 \mathrm{~mm} \mathrm{Hg})$ and standard therapy $(133.5 \mathrm{~mm}$ $\mathrm{Hg}$ ), the results showed no difference in overall CVD events after a follow-up of 4.7 years. However, the analysis of secondary endpoints showed a decrease in stroke $(\mathrm{p}=0.01)$ and macroalbuminuria $(\mathrm{p}=0.009)$ in the intensive BP group. It remains possible that the differential effect of low achieved BP on the latter secondary outcomes was due to more effective cerebral and renal autoregulation. It is important to note that subjects with serum creatinine $>1.5 \mathrm{mg} / \mathrm{dl}$ were excluded from AC$\mathrm{CORD}$, thus risk of progression to hard renal outcomes was low, as is the generalizability to the population seen in routine nephrology practices. Moreover, while about $40 \%$ of subjects in ACCORD had urine albumin levels $>30 \mathrm{mg} /$ day, data on outcomes by baseline level of albuminuria have not been reported. Moreover, the intensive therapy group had significantly higher rates of serious adverse events attributed to antihypertensive treatment, as well as higher rates of hypokalemia and elevations in serum creatinine level.

Nephron Clin Pract 2011;119:c324-c332 


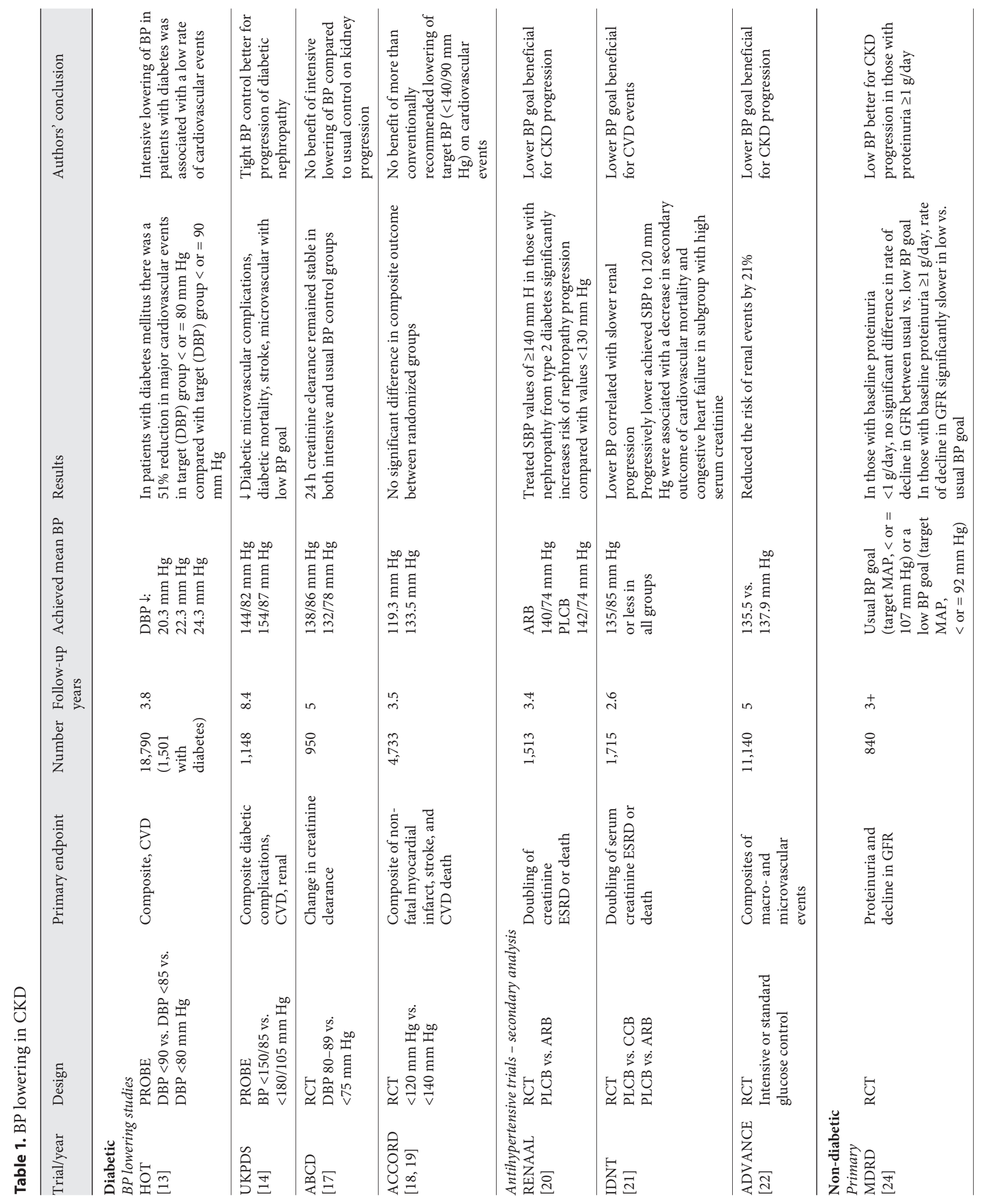




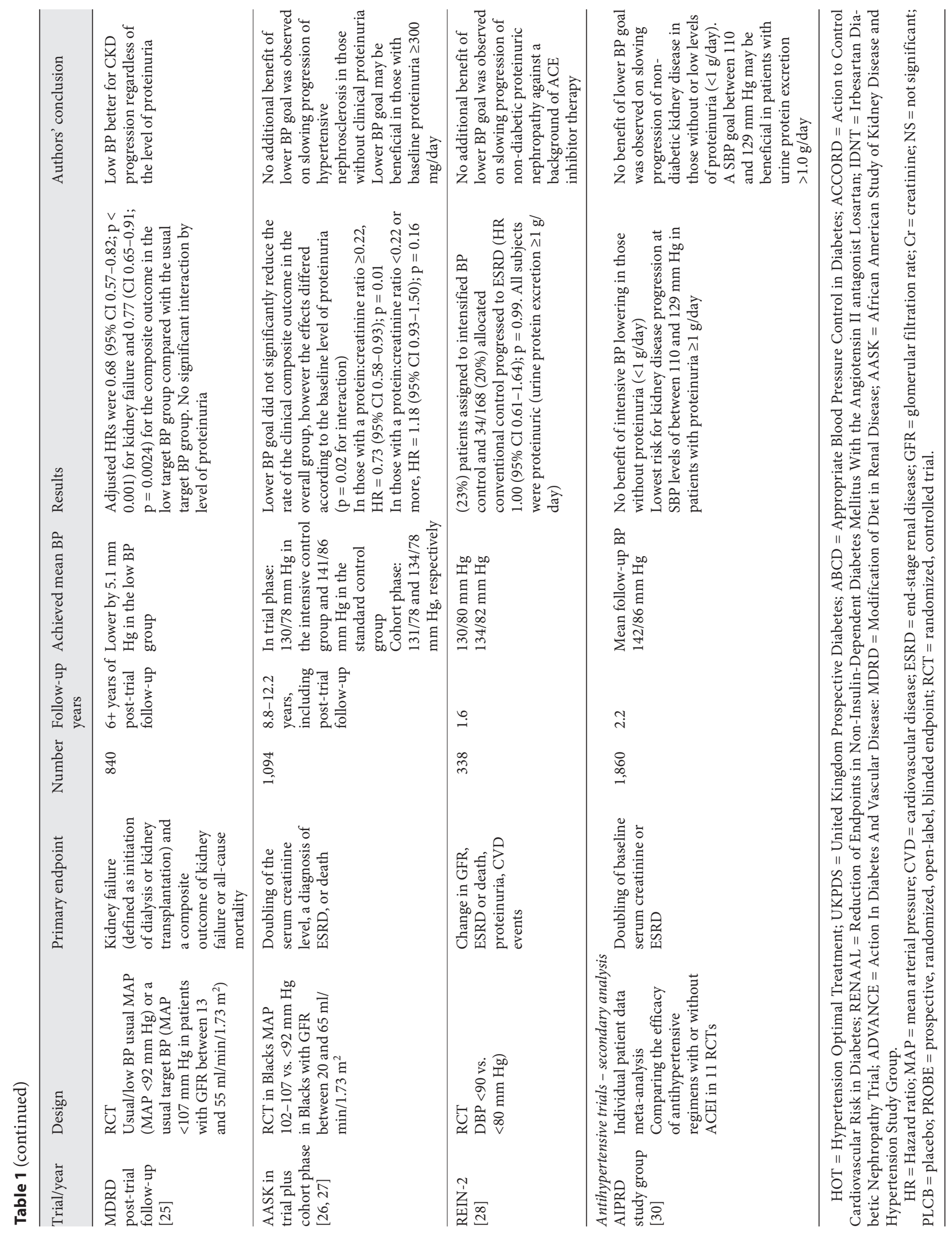


Evidence from secondary analysis of trials designed to study the impact of blockers of the renin-angiotensin system, including the Reduction of Endpoints in NIDDM with the Angiotensin II Antagonist Losartan (RENAAL) study and Irbesartan Diabetic Nephropathy Trial (IDNT) in patients with clinical proteinuria $(>500$ $\mathrm{mg} /$ day in RENAAL and $>900 \mathrm{mg} /$ day in IDNT) and type 2 diabetes, suggests that lower achieved SBP was more protective of kidney and CVD outcomes than higher levels $[20,21]$. The lowest risk was at lowest achieved BP, with no threshold level, suggesting that achieving $<120 / 80 \mathrm{~mm} \mathrm{Hg}$ would be advisable. Likewise, secondary analysis of the Action In Diabetes And Vascular Disease (ADVANCE) study on 11,140 patients aged 55 years or older with type 2 diabetes to undergo either standard glucose control or intensive glucose control with no prespecified BP targets, achieved a mean 5.6/2.2 $\mathrm{mm} \mathrm{Hg}$ greater reduction in BP, which resulted in a significant $9 \%$ reduction in relative risk of a major macro- or microvascular event and $21 \%$ reduction in renal events (new or worsening nephropathy), that is the development of macroalbuminuria, defined as a urinary albumin: creatinine ratio $>300 \mu \mathrm{g}$ of albumin per milligram of creatinine, or doubling of the serum creatinine level to at least $200 \mu \mathrm{mol} / \mathrm{l}(2.26 \mathrm{mg} / \mathrm{dl})$, the need for renal-replacement therapy, or death due to renal disease [22]. However, as patients were on active treatment of perindopril/ indapamide, it is debatable whether this effect was due to the renoprotective effect of ACE inhibitors or BP lowering only.

Thus, the overall weight of evidence from results of BP-lowering trials in type 2 diabetes indicates a possible benefit of intensive BP reduction based on results of UKPDS (in which BP goal was not intensive), subgroup analysis of diabetic subjects in HOT, or secondary analysis of data (UKPDS, ADVANCE). Clearly, these data are rather suboptimal for any firm recommendations to support target of $<130 / 80 \mathrm{~mm} \mathrm{Hg}$ in non-proteinuric diabetics, especially when primary outcomes of primary RCTs (ABCD, ACCORD) in with patients randomized to intensive versus conventional BP goals do not support benefit of kidney progression or cardiovascular outcomes. For diabetic patients with proteinuric CKD (>500 to $900 \mathrm{mg} /$ day), evidence from secondary analysis of RENAAL and IDNT suggests a possible benefit of lower targets (i.e. $<120 / 80 \mathrm{~mm} \mathrm{Hg}$ ). However, the relationship between $\mathrm{BP}$ and CKD is complex, and the issue of 'reverse causality' in secondary analysis always remains a high possibility - i.e. those less likely to progress due to non-BP related factors had lower BP, rather than intensive BP lowering leading to slower progression. Thus, future studies of BP targets in patients with type 2 diabetes must also stratify by the level of albuminuria for clearer guidance.

\section{BP Goal in Non-Diabetic CKD}

Three primary RCTs have evaluated target BP levels in patients with predominantly non-diabetic CKD [23]. In addition, evidence from secondary analysis of antihypertensive medications of achieved BP levels on CKD progression also provides some clues.

The Modification of Diet in Renal Disease (MDRD) study was a $2 \times 2$ factorial design, in which patients were randomly assigned to a low $(<125 / 75 \mathrm{~mm} \mathrm{Hg}$ ) or usual BP target and one of two types of diet with different levels of protein intake. During the main trial period of over 3 years, although there was no difference in risk of decline in GFR between the low and normal BP target in the overall groups, the low BP target was beneficial in the high proteinuria subgroup ( $>1 \mathrm{~g}$ ) [24]. However, it is worth noting that the effects of BP control in the MDRD study may have been partly confounded by the renoprotective effect of ACE inhibitors, which were taken by $54 \%$ of the patients in the low BP group, but only by $34 \%$ in the usual BP group. More recently, post-trial follow-up of the MDRD study participants over $6+$ years was performed in which the result for the hard outcome of kidney failure (defined as initiation of dialysis or kidney transplantation and a composite outcome of kidney failure or all-cause mortality) was favorable for the low BP target, for all patients regardless of the level of proteinuria [25]. However, the presence of a potential interaction between BP level and proteinuria on CKD progression, a categorical outcome of kidney failure, cannot be ruled out due to limited power, despite the longer duration of post-trial follow-up.

In the African American Study Kidney Disease (AASK) trial, 1,094 participants with mild to moderate chronic renal insufficiency (mean GFR $45.7 \mathrm{ml} / \mathrm{min}$ / $1.73 \mathrm{~m}^{2}$ ) caused by hypertension were divided into two groups with two BP interventions - mean arterial pressure (MAP) $<92 \mathrm{~mm} \mathrm{Hg}$ and MAP 102-107 mm Hg. Additionally, patients were randomized to one of three classes of antihypertensive agents - calcium channel blocker (amlodipine), converting enzyme inhibitor (ramipril), or $\beta$-blocker (metoprolol) [26]. Lower BP did not reduce the rate of GFR decline. After completing the trial phase of just over 4 years in which there was no dif- 
ference in decline in GFR by randomization to BP group, patients were invited to enroll in a cohort phase in which the $\mathrm{BP}$ target was $<130 / 80 \mathrm{~mm} \mathrm{Hg}$. In the cohort phase as well, there was no difference between the groups with a risk of primary outcome of rate of decline of the GFR slope. However, the effects during the total follow-up of 8.8-12.2 years on the composite outcome of doubling of the serum creatinine level, a diagnosis of end-stage renal disease (ESRD), or death, differed according to the baseline level of proteinuria, with a potential benefit in patients with a protein:creatinine ratio of $>0.22$ (300 $\mathrm{mg} / 24 \mathrm{~h}$ ). It is also important to note that despite good BP control, 53.9\% of the total AASK enrollees had reached an endpoint by the time of the report, indicating that progression of CKD and onset of CVD was faster in these subjects [27].

In the Ramipril Efficacy in Nephropathy-2 (REIN-2) study, participants with proteinuria $>1 \mathrm{~g} / \mathrm{day}$, all of whom were receiving ramipiril, an ACE inhibitor, were randomly assigned (169 in each group) to either 'conventional' (DBP $<90 \mathrm{~mm} \mathrm{Hg}$ ) or intensified $(<80 \mathrm{~mm} \mathrm{Hg}$ ) BP control [28]. The latter was aimed with additional therapy with felodipine, a dihydropyridine calcium channel blocker. The mean achieved SBP throughout follow-up was $129.6 \mathrm{~mm} \mathrm{Hg}$ (SD 10.9) in the intensified BP control group and $133.7 \mathrm{~mm} \mathrm{Hg}$ (SD 12.6) in the conventional control arm $(p=0.0019)$. About $23 \%$ of the patients assigned to intensified BP control and 20\% allocated to conventional control progressed to ESRD over 3 years, indicating no additional benefit of a lower BP goal with dihydropyridine calcium channel blocker in the presence of background treatment with ACE inhibitors. However, the study was powered to determine a $50 \%$ reduction in the primary outcome of cumulative incidence of ESRD - a rather large benefit. The expected benefit from a $5 \mathrm{~mm} \mathrm{Hg}$ decline in SBP is modest in magnitude. Moreover, background therapy with ACE inhibitors in this study already reduced the risk of progression of proteinuric nephropathy, and thus further reduction in risk from BP lowering would require a large sample size for determination of potential differential benefit. Thus, overall the REIN-2 results should be interpreted as rather inconclusive for a modest and significant benefit of aggressive BP reduction in proteinuric non-diabetic nephropathies.

A recent systematic review of three RCTs including the MDRD, AASK, and REIN-2 in adults with CKD did not conclusively show that the BP target of $<130 / 80 \mathrm{~mm} \mathrm{Hg}$ improves clinical outcomes more than the conventional target of $<140 / 90 \mathrm{~mm} \mathrm{Hg}$ in adults with CKD [29]. How- ever, the clinical heterogeneity for types of CKD, measures of proteinuria, and outcome definitions precluded a quantitative meta-analysis of these studies. Subjects in the low target BP groups needed more antihypertensive medications and had a slightly higher rate of adverse events. However, a lower target was potentially beneficial in persons with proteinuria $>0.31 \mathrm{~g} /$ day, for Blacks and non-Blacks, respectively.

Overall, these assessments are also consistent with secondary analysis of pooled data on 1,860 patients included in RCTs of antihypertensive regimens with versus without ACE inhibitors to slow progression of non-diabetic CKD [30]. The mean duration of follow-up was 2.2 years. SBP was more strongly correlated with kidney disease progression than DBP. A greater benefit of lower achieved BP (optimal level SBP 110-130 mm Hg) achieved during follow-up was associated with better renal outcomes (doubling of baseline serum creatinine or onset of ESRD) than higher levels of BP in patients with urine protein $\geq 1 \mathrm{~g} /$ day (interaction $\mathrm{p}<0.006$ ). At the same time, lowering BP below $110 \mathrm{~mm} \mathrm{Hg}$ was associated with acute kidney injury. These relationships were consistent in a number of sensitivity analyses using BP 6 months in advance, a mean of all $\mathrm{BP}$ readings during the entire period of follow-up, and from the same time as the outcome. No additional benefit of lower than conventional levels of achieved BP were observed in those with proteinuria $<1 \mathrm{~g} /$ day. Others have also reported a J-shaped association of SBP with greater risk of stroke at SBP $<120$ $\mathrm{mm} \mathrm{Hg}$ in patients with advanced stages (3 and 4) of CKD [10].

Nevertheless, reverse causation with low BP as a result of systemic illness resulting in multiple co-morbidities, rather than low BP leading to kidney disease progression or even acute kidney injury or stroke, would also yield similar associations between BP levels and adverse outcomes on secondary analysis. Hence, primary RCTs designed to study the cause-and-effect relationships, especially at lower levels of BP, are needed.

The results of the ongoing Systolic Blood Pressure Intervention Trial (SPRINT), a randomized, multicenter clinical trial on about 7,500 participants testing the effects of intensive lowering of SBP (goal of $<120$ vs. $<140$ $\mathrm{mm} \mathrm{Hg}$ ) on preventing CVD, will shed more light on the subject, especially since SPRINT aims to recruit at least 3,500 people with stage 3 CKD (estimated GFR of 30-59 $\mathrm{ml} / \mathrm{min} / 1.73 \mathrm{~m}^{2}$ ). The primary composite endpoints will be CVD mortality and non-fatal myocardial infarct, stroke, and heart failure. However, even the SPRINT study has not stratified the level of proteinuria, even in 
those with low GFR, albeit there may be power to detect a potential interaction between BP and clinical proteinuria on renal and CVD outcomes.

\section{Conclusions}

BP lowering is an important therapeutic goal in patients with diabetic and non-diabetic CKD. The spectrum of the disease is wide, with benefit of strategies influenced by patient characteristics that determine the rate of progression and onset of CVD. However, evidence on $\mathrm{BP}$ reduction that accounts for the heterogeneity of clinical presentation is rather scarce. In diabetic kidney disease, while data support lowering BP to levels $<140 / 90 \mathrm{~mm} \mathrm{Hg}$, evidence in favor of pushing the levels to $<130 / 80 \mathrm{~mm} \mathrm{Hg}$ is largely driven by subgroup (HOT) or secondary analysis (UKPDS, ADVANCE), and primary RCT data do not favor further intensive targets (i.e. $<120 / 80 \mathrm{~mm} \mathrm{Hg}$ ) in all subjects with diabetes (ABCD, ACCORD). More research is needed to determine whether a lower $\mathrm{BP}$ target confers additional benefit to diabetic patients in the presence of clinical albuminuria, especially since secondary analyses are suggestive (IDNT, RENAAL) that such a goal might be associated with additional benefit.

Thus, aiming for a BP of $<130 / 80 \mathrm{~mm} \mathrm{Hg}$ in patients with diabetic CKD may be justifiable, especially in the presence of clinical proteinuria of $\geq 1 \mathrm{~g} /$ day.

In patients with non-diabetic CKD without albuminuria, the weight of evidence for BP target is in favor of $<140 / 90 \mathrm{~mm} \mathrm{Hg}$. For non-diabetic CKD with albuminuria, evidence suggests different cut-off levels to stratify albuminuria for BP goals $\geq 300 \mathrm{mg} /$ day in Blacks or $\geq 1 \mathrm{~g} /$ day in non-Blacks, and aiming for a target BP of $<130 / 80 \mathrm{~mm} \mathrm{Hg}$ at these levels would be reasonable. However, in all patients with CKD, it is best to avoid reduction of SBP to levels $<110 \mathrm{~mm} \mathrm{Hg}$ since there has been no benefit documented with such levels of intensive lowering even in secondary analysis; on the contrary, there may be risk of harm with such an aggressive approach.

Clearly, evidence-based medicine aims to apply the best available scientifically collected evidence to arrive at clinical decision-making. Thus, consistent results from adequately powered and well-conducted RCTs randomizing patients with key characteristics relevant to the clinical practice of nephrology (diabetic and non-diabetic CKD with different levels of albuminuria, kidney function, and race) and to BP targets for hard kidney and cardiovascular outcomes would provide the highest level of evidence for clinical practice guidelines. Since conclusive evidence from RCT is limited for the entire spectrum of patients encountered by nephrologists, subgroup and secondary analysis provide useful information for the outcomes of interest albeit recognizing that individual judgment and caution be exercised with strict regimens, especially where the strength of evidence is less than optimal.

It is therefore important to underscore that there is clearly a need for well-designed RCTs stratified by patient characteristics such as albuminuria and race to determine the optimal level of BP for slowing the progression of CKD and preventing CVD in patients with diabetic and non-diabetic kidney disease. It is also vital that in addition to serious adverse events, information on quality of life and cost effectiveness be also collected for a comprehensive assessment from the patients' and healthplanners' perspectives.

\section{References}

1 Bello AK, Nwankwo E, El Nahas AM: Prevention of chronic kidney disease: a global challenge. Kidney Int Suppl 2005;S11-S17.

$\checkmark 2$ Coresh J, Selvin E, Stevens LA, et al: Prevalence of chronic kidney disease in the United States. JAMA 2007;298:2038-2047.

$\checkmark 3$ Stevens LA, Coresh J, Greene T, Levey AS: Assessing kidney function - measured and estimated glomerular filtration rate. N Engl J Med 2006;354:2473-2483.

4 Tuomilehto J, Rastenyte D, Birkenhager WH, et al: Effects of calcium-channel blockade in older patients with diabetes and systolic hypertension. Systolic Hypertension in Europe Trial Investigators. N Engl J Med 1999;340:677-684.
SHEP Cooperative Research Group: Prevention of stroke by antihypertensive drug treatment in older persons with isolated systolic hypertension. Final results of the Systolic Hypertension in the Elderly Program (SHEP). JAMA 1991;265:3255-3264.

$\checkmark 6$ Wang JG, Staessen JA, Gong L, Liu L: Chinese trial on isolated systolic hypertension in the elderly. Systolic Hypertension in China (Syst-China) Collaborative Group. Arch Intern Med 2000;160:211-220.

7 Dahlof B, Lindholm LH, Hansson L, Schersten B, Ekbom T, Wester PO: Morbidity and mortality in the Swedish Trial in Old $\mathrm{Pa}$ tients with Hypertension (STOP-Hypertension). Lancet 1991;338:1281-1285.
8 Multiple Risk Factor Intervention Trial Research Group: Multiple risk factor intervention trial. Risk factor changes and mortality results. JAMA 1982;248:1465-1477.

$>9$ Bakris GL, Williams M, Dworkin L, et al: Preserving renal function in adults with hypertension and diabetes: a consensus approach. National Kidney Foundation Hypertension and Diabetes Executive Committees Working Group. Am J Kidney Dis 2000;36: 646-661.

10 Weiner DE, Tighiouart H, Levey AS, et al: Lowest systolic blood pressure is associated with stroke in stages 3 to 4 chronic kidney disease. J Am Soc Nephrol 2007;18:960966. 
-11 Little WC, Zile MR, Klein A, Appleton CP, Kitzman DW, Wesley-Farrington DJ: Effect of losartan and hydrochlorothiazide on exercise tolerance in exertional hypertension and left ventricular diastolic dysfunction. Am J Cardiol 2006;98:383-385.

-12 Parving HH, Andersen AR, Smidt UM, Svendsen PA: Early aggressive antihypertensive treatment reduces rate of decline in kidney function in diabetic nephropathy. Lancet $1983 ; 1: 1175-1179$.

$\checkmark 13$ Hansson L, Zanchetti A, Carruthers SG, et al: Effects of intensive blood pressure lowering and low-dose aspirin in patients with hypertension: principal results of the Hypertension Optimal Treatment (HOT) randomised trial: HOT study group. Lancet 1998;351:1755-1762.

14 UK Prospective Diabetes Study Group: Tight blood pressure control and risk of macrovascular and microvascular complications in type 2 diabetes: UKPDS 38. BMJ 1998;317: 703-713.

15 Adler AI, Stratton IM, Neil HA, et al: Association of systolic blood pressure with macrovascular and microvascular complications of type 2 diabetes (UKPDS 36): prospective observational study. BMJ 2000;321:412-419.

-16 Chobanian AV, Bakris GL, Black HR, Cushman WC, Green LA, Izzo JL Jr, Jones DW, Materson BJ, Oparil S, Wright JT Jr, Roccella EJ; National Heart, Lung, and Blood Institute Joint National Committee on Prevention, Detection, Evaluation, and Treatment of High Blood Pressure; National High Blood Pressure Education Program Coordinating Committee: The Seventh Report of the Joint National Committee on Preven- tion, Detection, Evaluation, and Treatment of High Blood Pressure: the JNC 7 report. JAMA 2003;289:2560-2572.

$\checkmark 17$ Schrier RW, Estacio RO, Mehler PS, Hiat WR: Appropriate blood pressure control in hypertensive and normotensive type 2 diabetes mellitus: a summary of the ABCD trial. Nat Clin Pract Nephrol 2007;3:428-438.

18 Cushman WC, Evans GW, Byington RP, et al: Effects of intensive blood pressure control in type 2 diabetes mellitus. N Engl J Med 2010;362:1575-1585.

19 Riddle MC: Effects of intensive glucose lowering in the management of patients with type 2 diabetes mellitus in the Action to Control Cardiovascular Risk in Diabetes (ACCORD) trial. Circulation 2010;122:844846.

20 Brenner BM, Cooper ME, de Zeeuw D, et al: Effects of losartan on renal and cardiovascular outcomes in patients with type 2 diabetes and nephropathy. N Engl J Med 2001;345: 861-869.

21 Lewis EJ, Hunsicker LG, Clarke WR, et al: Renoprotective effect of the angiotensin-receptor antagonist irbesartan in patients with nephropathy due to type 2 diabetes. N Engl J Med 2001;345:851-860.

22 Patel A, MacMahon S, Chalmers J, et al: Intensive blood glucose control and vascular outcomes in patients with type 2 diabetes. $\mathrm{N}$ Engl J Med 2008;358:2560-2572.

23 Klahr S, Levey AS, Beck GJ, et al: The effects of dietary protein restriction and blood pressure control on the progression of chronic renal disease. Modification of Diet in Renal Disease Study Group. N Engl J Med 1994; 330:877-884.
24 Peterson JC, Adler S, Burkart JM, et al: Blood pressure control, proteinuria, and the progression of renal disease. The Modification of Diet in Renal Disease study. Ann Intern Med 1995;123:754-762.

25 Sarnak MJ, Greene T, Wang X, et al: The effect of a lower target blood pressure on the progression of kidney disease: long-term follow-up of the Modification of Diet in Renal Disease study. Ann Intern Med 2005;142: 342-351.

26 Wright JT Jr, Bakris G, Greene T, et al: Effect of blood pressure lowering and antihypertensive drug class on progression of hypertensive kidney disease: results from the AASK trial. JAMA 2002;288:2421-2431.

27 Appel LJ, Wright JT Jr, Greene T, et al: Intensive blood pressure control in hypertensive chronic kidney disease. N Engl J Med 363: 918-929.

28 Ruggenenti P, Perna A, Loriga G, et al: Blood pressure control for renoprotection in patients with non-diabetic chronic renal disease (REIN-2): multicentre, randomised controlled trial. Lancet 2005;365: 939-946.

29 Upadhyay A, Earley A, Haynes SM, Uhlig K: Systematic review: blood pressure target in chronic kidney disease and proteinuria as an effect modifier. Ann Intern Med 2011;154: 541-548.

30 Jafar TH, Stark PC, Schmid CH, et al: Progression of chronic kidney disease: the role of blood pressure control, proteinuria, and angiotensin-converting enzyme inhibition: a patient-level meta-analysis. Ann Intern Med 2003;139:244-252.

\title{
Editorial Comment
}

\author{
Meguid El Nahas, Sheffield
}

The minireview by A. Faqah and T.H. Jafar exposes the issues related to BP target values in CKD. Numerous statements suggested that the lower the BP, the slower the progression of CKD. A number of guidelines have stipulated target values around 130/80 $\mathrm{mm} \mathrm{Hg}$ and even lower in patients with diabetes and those with proteinuria. The evidence upon which these guidelines were based relied primarily on observational data as well as secondary or post hoc analyses of clinical trials and systematic and meta-analyses of heterogeneous data. This review exposes the weakness of such evidence. As so often in nephrology, evidence is gathered from observational studies that remain unsubstantiated by subsequent attempts at confirmation by RCTs. So often in nephrology, evidence is sought from secondary and post hoc analyses not intended or powered to answer the question raised...! So often in nephrology, dogma prevails enforced by unsubstantiated guidelines...!

Most RCTs mentioned in the review that attempted to answer whether intensive BP control is beneficial in slowing the progression of CKD failed to show a significant difference in renal functional outcomes. Furthermore, 
concern about harm has been raised in those submitted to intensive treatment with increased morbidity and mortality.

Finally, the assumption that one size fits all with target systolic levels of $<130$ or $120 \mathrm{~mm} \mathrm{Hg}$ is preposterous. Age, race and comorbidities may dictate different target levels. This is becoming apparent in patients on hemodialysis where older and diabetic individuals had higher mortality rates at lower $(<140 / 70 \mathrm{~mm} \mathrm{Hg}) \mathrm{BP}$ levels. More remarkable was the observation in this cohort analysis that survival did not seem to be harmed by higher systolic and diastolic values [1]. In conclusion, it is high time that $\mathrm{BP}$-related guidelines in $\mathrm{CKD}$ are reviewed; this is currently underway by KDIGO. It is also high time that nephrologists base their practice on substantiated guidelines.... It is also high time that nephrologists critically appraise the data submitted to them by 'experts' and refute assertions based on secondary and post hoc analyses as weak at best. We have a long way to go!

\section{Reference}

Myers OB, Adams C, Rohrscheib MR, Servilla KS, Miskulin D, Bedrick EJ, Zager PG: Age, race, diabetes, blood pressure, and mortality among hemodialysis patients. J Am Soc Nephrol 2010;21:1970-1978. 